\title{
Aphakic macular oedema following prosthetic lens implantation*
}

\author{
DAVID EASTY, NEIL DALLAS, AND RONAN O'MALLEY \\ From the Bristol Eye Hospital, Lower Maudlin Street, Bristol
}

SUMMARY Fluorescein angiography of the iris was performed on patients with plastic lens implants with cystoid oedema of the macula, and the nature of the vascular changes was compared with controls provided by patients who did not have macular disease. Densitometry was used to quantitate leakage of fluorescein dye into the anterior chamber in residual angiograms 5 minutes after injection. Cystoid oedema was associated with marked increase in vascular permeability of the iris in the patients with implants, and in aphakics without implants, but to a lesser degree. Although it cannot be concluded that implants themselves cause either the increase in permeability or the onset of macular oedema, the presence of such implants must be considered an additional hazard in an eye which is already at risk. Iris angiography can be used as a diagnostic aid in patients in whom macular oedema is suspected. A persistent increase in permeability associated with a reduction in visual acuity should act as a warning of further visual loss and of eventual cystoid macular degeneration.

With the increasing use of prosthetic lens implantation following cataract extraction, the nature and incidence of operative and postoperative complications are being investigated in more depth. Postoperative anterior uveitis, retinal detachment, acrylic lens dislocation, glaucoma, bullous keratopathy, and cystoid macular oedema have all been reported, but it is the macular disease which is particularly a cause for disappointment to the patient and concern for the ophthalmic surgeon.

Macular oedema and visual loss following cataract extraction were originally described by Irvine (1953) as being associated with irritability of the eye and also rupture of the anterior hyaloid membrane leading to vitreous strands adhering to the section. Gass and Norton (1969) stated that adherence and traction were not a necessity for the development of macular oedema but when present tended to worsen the condition. In a study of macular disturbance 6 weeks after cataract extraction (Hitchings et al., 1975) transient oedema occurred in $50 \%$ of patients examined. No such changes developed in a group of controls who had glaucoma surgery. The authors

*Paper presented at the meeting of the Ophthalmic Section of the Royal Society of Medicine in Bristol on 13 May 1976

Address for reprints: D. L. Easty, FRCS, Bristol Eye Hospital, Lower Maudlin Street, Bristol BS1 2LX, Avon considered the major differences between the two groups were the actual cataract extraction and the presence of postoperative vitreous inflammation. Assessment 6 months after cataract extraction showed that $10 \%$ had a corrected visual acuity of $6 / 12$ or $6 / 18$, while in the remainder the acuity was $6 / 9$ or better. Cataract extraction may frequently induce macular oedema, which generally resolves but in a small proportion of cases leads to a varying degree of visual loss.

The incidence of cystoid oedema of the macula in pseudophakia has been variously stated to be $5 \%$ (Pearce, 1972), 12\% (Binkhorst and Leonard, 1967), $19 \%$ (Jardine and Sandford-Smith, 1974), and 7\% (Dallas, 1976), which are all greater than the $2 \%$ incidence following plain intracapsular cataract extraction in a series of 200 patients. Since the frequency and severity of cystoid macular oedema are known to be greater in patients with implants than in aphakic patients without implants, the question arises whether the implant is responsible.

Iris angiography has frequently been used to assess the state of the iris vasculature in a number of diseases (Mitsui et al., 1969; Bron and Easty, 1970; Vannas, 1969; Cobb et al., 1970; Easty and Chignell, 1973). In a group of 9 patients with cystoid macular oedema after intracapsular cataract extraction moderate to massive leakage of fluorescein dye 
was noted on iris angiography (Kottow and Hendrickson, 1975). In order to determine how the iris vasculature is affected by acrylic lens implants, and its possible relation to cystoid macular oedema, iris fluorescein angiography was performed on groups of patients with and without macular oedema.

Prostaglandins are known to act as one of the mediators for the responses of the eye to trauma (Ambache et al., 1965; Cole and Ungar, 1973). Iris-suspended lenses are not perfectly stable. Because of this they may cause mechanical stimulation, which could produce detectable increases in pharmacological mediators in the intraocular fluids, which in turn may play a role in the production of macular disease. As a supplementary study, therefore, prostaglandin-like activity was sought in aqueous humour taken from patients with cystoid macular oedema who were undergoing further operative procedures. Experimental studies were also performed on aqueous humour taken from rabbits from which the crystalline lenses had been removed and replaced with acrylic implants.

\section{Materials and methods}

Iris fluorescein angiography studies were performed on 40 patients after cataract extraction at least 6 months previously, with and without signs of cystic macular oedema and with or without acrylic implants (Table 1). Acrylic lens implantation was performed by one of us (N.L.D.) using Binkhorst ( 8 eyes) or Federov iris-clip lens (21 eyes) or the Ridley anterior chamber implant (12 eyes, also referred by Mr C. A. Brown). A full ocular examination was carried out on all patients prior to the study. The intraocular pressures were measured and the state of the iris and the anterior chamber was examined. The retina and vitreous were examined in detail with the slit-lamp microscope. It was not possible to use retinal fluorescein angiographic photography to determine whether the signs were typical of cystoid macular degeneration, because pupils could not be safely dilated with mydriatics; however, confirmatory evidence was obtained with indirect ophthalmoscopy fitted with appropriate filters after the intravenous injection of fluorescein dye. In all affected patients there had been good postoperative visual recovery, though this was followed by visual deterioration some months later. No cause other than macular oedema was found to explain the loss of vision.

Iris angiography was performed by a standard technique (Easty and Bron, 1971) in patients not less than 6 months after operation. Photographs were taken at 2-second intervals for periods of 30 to 60 seconds, and residual photographs were taken after
Table 1 Case selection for iris fluorescein angiography

\begin{tabular}{|c|c|}
\hline & Number of eyes \\
\hline \multicolumn{2}{|l|}{ Controls } \\
\hline Phakic & 29 \\
\hline Aphakic: without macular oedema & 7 \\
\hline with macular oedema & 4 \\
\hline \multicolumn{2}{|l|}{ Aphakics with implants } \\
\hline Iris clip without macular oedema & 14 \\
\hline Iris clip with macular oedema & 15 \\
\hline \multicolumn{2}{|l|}{ Ridley implant without macular oedema 8} \\
\hline Ridley implant with macular oedema & 4 \\
\hline
\end{tabular}

an interval of 5 minutes. Ilford FP4 film was processed according to a standard method using developer (Qualitol, May \& Baker) for 10 minutes at a dilution of 1 in 20 at $68^{\circ} \mathrm{F}\left(20^{\circ} \mathrm{C}\right)$. Contact sheets were routinely printed (Ilford PQ Universal Developer for 2 minutes at 1 in 9 dilution at $68^{\circ} \mathrm{F}$ $\left.\left(20^{\circ} \mathrm{C}\right)\right)$. The iris angiograms were examined for signs of vascular abnormality and particularly for leakage of fluorescein into the iris stroma or anterior chamber. The magnitude of the fluorescence that occurred was measured directly from the angiogram with the aid of a standard densitometer.

Prostaglandin assays were performed on aqueous humour taken from 4 patients all of whom had plastic implants and 3 of whom had cystoid macular oedema. Serial measurements were also performed on fluid taken from the anterior chambers of 4 rabbits, 2 having had plastic implants following lens removal, and 2 having had simple removal of the lens. Samples were extracted for prostaglandins with ethanol acidified with formic acid followed by chloroform. They were then assayed against PGE 2 for prostaglandin-like activity on rat stomach strips (Vane, 1957) suspended in Krebs' solution at $37^{\circ} \mathrm{C}$.

\section{Results}

IRIS ANGIOGRAPHY IN THE IMMEDIATE

POSTOPERATIVE PERIOD

Postoperative angiographic studies showed that patchy leakage of fluorescein occurred into the iris stroma from dilated radial vessels in all patients investigated, and this was then followed by diffusion of dye into the aqueous humour. The changes occurred both in the presence and in the absence of an implant. The leakage persisted for between 6 weeks and 3 months and then was followed by a return to a normal angiographic appearance.

ANGIOGRAPHY FOLLOWING INTRACAMERAL IMPLANTS WITHOUT MACULAR OEDEMA In the absence of macular oedema patients with intracameral implants showed no evidence of a disturbance in the vascular physiology of the iris when examined by fluorescein angiography. 


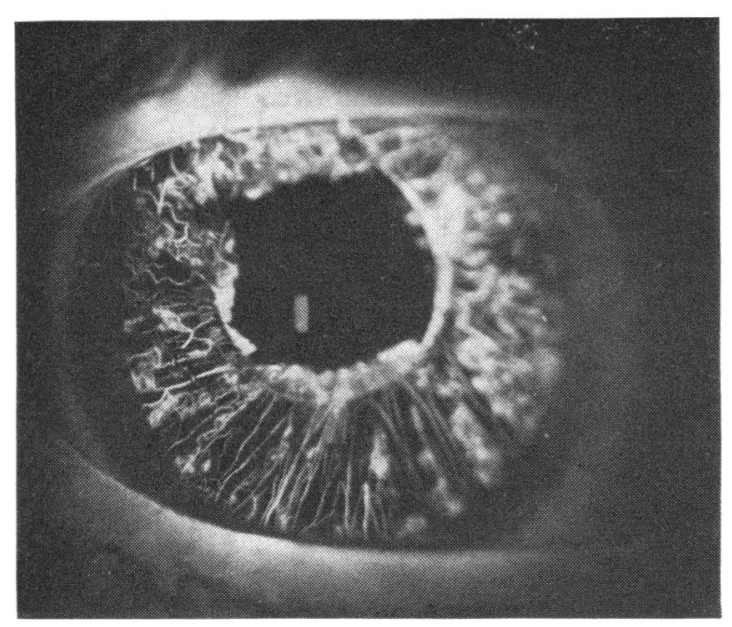

a

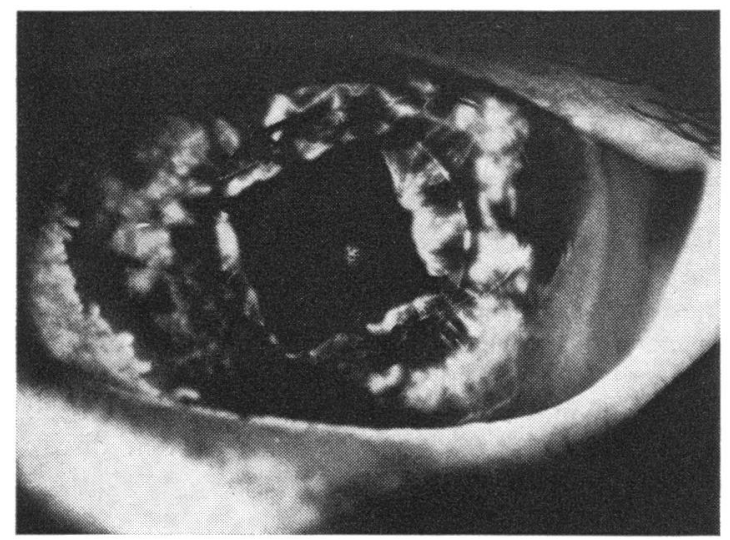

C

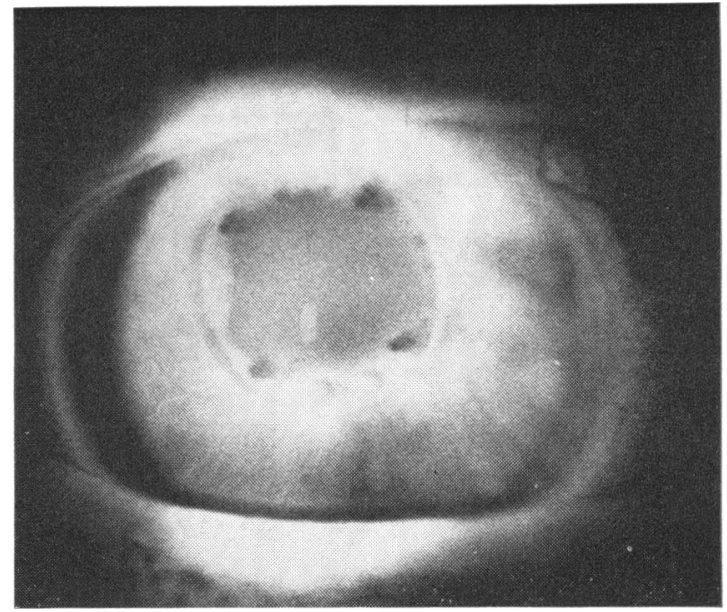

b

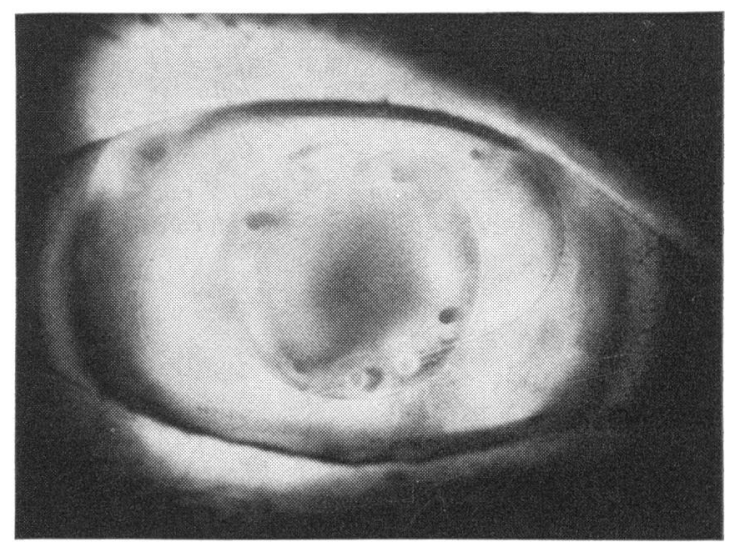

d

Fig. 1 (a and c) Iris angiograms 20 seconds after injection demonstrating vascular dilatation and early leakage in 2 patients with iris-clip implants and cystoid macular oedema; (b and d) Residual angiograms in the same patients 5 minutes after injection, showing pronounced leakage into the anterior chambers

ANGIOGRAPHY FOLLOWING INTRACAMERAL IMPLANTS WITH CYSTOID MACULAR OEDEMA Nineteen patients had clinical evidence of cystoid macular disease; of these 14 had massive leakage of fluorescein from the iris vessels, 4 had marked leakage, and 1 had only moderate leakage. Fig. 1 shows the appearance that was found in 2 patients who had developed cystoid macular oedema, in both of whom the postoperative visual acuity had been good though it was subsequently followed by visual deterioration. During the early transit of the dye iris vessels are notably dilated, and there is leakage into the iris stroma. Residual angiograms show that there is also diffusion into the anterior chamber, which masks the fluorescing vessels of the iris.
CYSTOID MACULAR OEDEMA IN APHAKICS WITHOUT LENS IMPLANTS

Though in 4 patients in whom macular oedema had been confirmed by retinal angiography there was a moderate leakage into the iris stroma and anterior chamber, it was not so severe as when macular disease was accompanied by lens implantation.

\section{DENSITOMETRY}

Densitometric measurement of the residual fluorograms 5 minutes after injection showed that patients with cystoid macular degeneration had marked fluorescence in their anterior chambers, whereas the iris vasculature in patients without macular disease showed no difference from the vasculature in the normal or aphakic eye (Fig. 2). Iris colour did not 


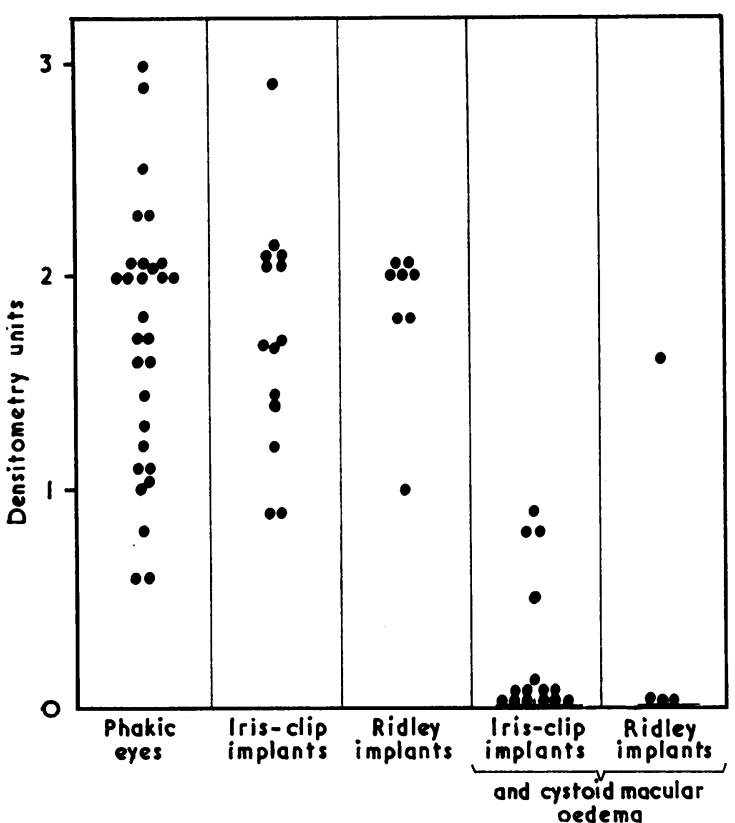

Fig. 2 Densitometry measurements of the residual iris angiograms in patients with cystoid macular oedema, compared with controls

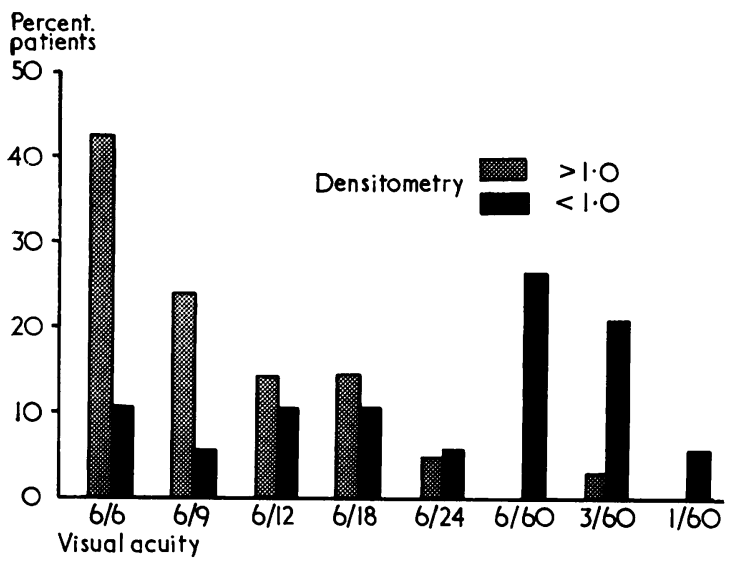

Fig. 3 Comparison of percentage distributions of visual acuity in 2 groups of patients; those with densitometry $>1.0$, and those with measurements $<1.00$

show correlation with the degree of fluorescein leakage in any of this group.

Fig. 3 demonstrates the distribution of visual acuity in two groups of patients-those with readings greater than unity (who had mild to moderate leakage) and those with readings less than unity (where the amount of leakage of dye was marked). Twenty $95 \%$ ) of 21 patients with mild to moderate leakage had a visual acuity equal to or better than $6 / 24$, while in 20 patients with severe leakage $58 \%$ had visual acuities equal to or less than $6 / 60(\mathrm{P}<0.01$, Fisher's exact probability test).

\section{PROSTAGLANDIN ASSAYS}

Assays for prostaglandin E1 and E2 in aqueous humour taken from 3 patients with macular oedema before operations on the same eye did not show raised levels. Similarly, assays performed on aqueous humour taken from two Dutch white rabbits with Federov implants showed no elevation when compared with levels in two controls in which only the crystalline lenses had been removed. The measurements were made 6 weeks after the operations.

\section{Discussion}

The results of the survey suggest that cystoid macular oedema in patients with a prosthetic lens implant is associated with persistent dilatation and increased permeability of the iris vessels. Cystoid macular disease is both more severe and more prevalent in these patients, and it seems possible that it could be the apposition of the implant to the iris vessels that may be indirectly responsible.

Many factors have been implicated in the causation of persistent aphakic macular oedema (Table 2). Vitreous complications during surgery are a known precipitating factor, as are the lens removal itself, postoperative vitritis, and systemic vascular disease (Hitchings et al., 1975).

Table 2 Possible influences in the pathogenesis of cystoid macular oedema

\begin{tabular}{|c|c|}
\hline \multicolumn{2}{|l|}{ In aphakia } \\
\hline Host factors: & $\begin{array}{l}\text { age, sex, occupation, systemic disease } \\
\text { (diabetes mellitus) }\end{array}$ \\
\hline Ocular factors: & $\begin{array}{l}\text { aetiology of cataract, associated disease } \\
\text { (retinal vein occlusion, uveitis), previous } \\
\text { surgery, innate predisposition }\end{array}$ \\
\hline Surgical factors: & $\begin{array}{l}\text { type of operation (intra- or extra-capsular), } \\
\text { technique, complications (e.g., vitreous loss } \\
\text { or inclusion in section, etc.) }\end{array}$ \\
\hline \multicolumn{2}{|c|}{ Postoperative factors: $\mathrm{u}$} \\
\hline Therapeutic: & topical adrenaline drops \\
\hline \multicolumn{2}{|c|}{ In aphakia following plastic lens implant } \\
\hline Surgical factors: & increased intraocular manipulation \\
\hline \multicolumn{2}{|c|}{ Postoperative factors: dislocation, uveitis, further surgery } \\
\hline $\begin{array}{l}\text { Factors related to } \\
\text { the implant: }\end{array}$ & $\begin{array}{l}\text { method of sterilisation } \\
\text { iris-clip movement } \\
\text { toxicity of plastic } \\
\text { antigenicity of plastic }\end{array}$ \\
\hline
\end{tabular}


Plastic implants could provide a superadded factor which might affect the macula in a number of ways. Among these should be considered the influence of recurrent episodes of minor trauma, particularly in iris-supported lenses, the toxicity of the component parts, and the antigenicity of these parts.

In the patients with cystoid macular oedema operative and immediate postoperative complications were not a factor. It is conceivable that the continuing wobble in iris-supported lenses may stimulate neurogenic reflexes which would result in vasodilatation of the iris vessels, with a similar response in the ciliary body and elsewhere. At the same time such minor trauma could result in the production of pharmacologically active amines-for example, histamine, 5-hydroxytryptamine, bradykinin, and prostaglandin-which would also act to maintain the vessels in a state of increased permeability and might in themselves be significant in the production of macular oedema. However, bioassay of one of these substances (prostaglandin) in anterior chamber fluid taken from patients with cystoid oedema undergoing further operative procedures following lens implantation did not show a rise in level.

Implants are composed of polymethylmethacrylate, which does not undergo depolymerisation, in contrast to some other plastics, monomer breakdown products of which produce toxic sequelae in eyes. The lack of toxicity is supported by studies carried out by Galin et al. (1975), in which cell cultures were not affected by the presence of the plastic, in contrast to which cells failed to grow or showed cytopathic effects in the presence of the polyamide loops.

One way in which plastic implants may create inflammation is by an immunological route, for with the breakdown of the blood-aqueous barrier in the vessels of the iris the anterior chamber is no longer a privileged site. Acrylic materials are known to be good skin sensitisers, and it is possible that both cell-mediated and humoral immunity may be involved in the reaction. It is the humoral response which results in production of such mediators of inflammation as histamine, slow-reacting substance, serotonin, prostaglandins, kinins, lysosomal enzymes, and neurotrophic polypeptides. Certainly an immunological reaction is suggested in the occasional patient when there is a preferential adherence of inflammatory cells to the implant forming precipitates, which are probably lymphocytes or macrophages. It is in these cases that keratic precipitates do not necessarily form. We have noted, however, that in patients showing these reactions there is no evidence of cutaneous hypersensitivity when they are patch-tested to acrylic materials, neither do they demonstrate antigen-stimulated in vitro lymphocyte transformation.

The production of cystoid macular oedema in patients with lens implants is therefore multifactorial, but probably the lens itself is a contributing factor. The possible relation of minor trauma to the iris diaphragm is suggested by the fact that preliminary results of iridocapsular implants, where such trauma would be less severe, show a reduction in its incidence. Although the plastic materials used are unlikely to be toxic, because they are known to be antigenic an immunological mechanism remains a possibility.

Florescein angiography of the iris is of value in assessing the response of the ocular tissues to implants and can be of help in the diagnosis of macular disease in its preliminary stages. We are routinely examining postoperative patients by this method, and in cases of persistently increased vascular permeability associated with variable visual acuity early removal of the lens is considered as a preventive measure to reduce the visual loss caused by persistent macular oedema.

We sincerely thank Mr Christopher Dean Hart and Mr Anthony Makepiece for their advice and help, and Mr J. Morgan for photographic assistance. We acknowledge the help of Mr C. A. Brown, who allowed us to examine his patients. We are grateful to Dr Alan Rogers and his staff, who kindly estimated prostaglandin levels in aqueous humour.

\section{References}

Ambache, N., Kavanagh, L., and Whiting, J. (1965). Effect of mechanical stimulation of rabbit's eyes: release of active substance in anterior chamber perfusates. Journal of Physiology, 176, 378-407.

Binkhorst, C. D., and Leonard, P. A. M. (1967). Results in 208 iris-clip pseudophakos implantations. American Journal of Ophthalmology, 64, 947-956.

Bron, A. J., and Easty, D. L. (1970). Fluorescein angiography of the globe and anterior segment. Transactions of the Ophthalmological Societies of the United Kingdom, 90, 339367.

Cobb, B., Shilling, J. S., and Chisholm, I. H. (1970). Vascular tufts at the pupillary margin in myotonic dystrophy. American Journal of Ophthalmology, 69, 573-582.

Cole, D. F., and Ungar, W. G. (1973). Prostaglandins as mediators for the responses of the eye to trauma. Experimental Eye Research, 17, 357-368.

Dallas, N. L. (1976). Comparison of iris clip lens results with plain intracapsular extraction. Proceedings of the Royal Society of Medicine, 69, 903-905.

Easty, D. L., and Chignell, A. H. (1973). Fluorescein angiography in anterior segment ischaemia. British Journal of Ophthalmology, 57, 18-26.

Easty, D. L., and Bron, A. J. (1971). Fluorescein angiography of the anterior segment. British Journal of Ophthalmology, $55,671-682$.

Galin, M. A., Chowchuvech, E., and Galin, A. (1975). 
Tissue culture methods for testing the toxicity of ocular plastic materials. American Journal of Ophthalmology, 79, 665-669.

Gass, J. D. M., and Norton, E. W. D. (1969). Follow-up study of cystoid macular oedema following cataract extraction. Transactions of the American Academy of Ophthalmology and Otolaryngology, 73, 665-682.

Hitchings, R. A., Chisholm, I. H., and Bird, A. C. (1975). Aphakic macular oedema: incidence and pathogenesis. Investigative Ophthalmology, 14, 68-72.

Irvine, S. R. (1953). A newly defined vitreous syndrome following cataract surgery. American Journal of Ophthalmology, 37, 599-619.

Jardine, P., and Sandford-Smith, J. H. (1974). Federov irissupported intraocular acrylic lens. British Journal of Ophthalmology, 58, 718-724.
Kottow, M., and Hendrickson, P. (1975). Iris angiography in cystoid macular oedema after cataract extraction. Archives of Ophthulmology, 93, 487-493.

Pearce, J. L. (1972). Long-term results of the Binkhorst iris clip lens in senile cataract. British Journal of Ophthalmology, 56, 319-331.

Mitsui, Y., Matsubara, M., and Kanagawa, M. (1969). Fluorescence irido corneal photography. British Journal of Ophthalmology, 53, 505-512.

Vannas, A. (1969). Fluorescein angiography of the vessels of the iris in pseudo-exfoliation of the lens capsule, capsular glaucoma and some other forms of glaucoma. Acta Scandinavica Ophthalmologica, 105, 28-75.

Vane, J. R. (1957). A sensitive method for assay of 5 hydroxytryptamine. British Journal of Pharmacology, 12, 344-349. 